\title{
Review article
}

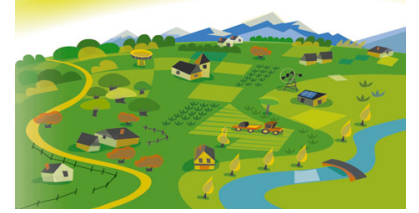

\section{Digital imaging information technology applied to seed germination testing. A review}

\author{
Antonio DeLL' AQUILA* \\ Via Abate Gimma 247, 70122 Bari, Italy
}

(Accepted 19 June 2008)

\begin{abstract}
The application of digital imaging information technology to seed germination testing is discussed. This technology is reviewed in light of recent interest on the development and adoption of sustainable agrosystems joined with a modern strategy of "precision agriculture", which provides new complex information tools for better crop production. Basic concepts on the patterns of image analysis descriptors of imbibing seed performance are described with the objective of demonstrating the potential of this technique to be adequate for overcoming problems encountered with a standard seed germination test. The application of different image analysis system prototypes in monitoring seed germination of Brassica, as well as several other crop species, has provided encouraging results, highlighting the reliability of this technique to quickly acquire digital images and to extract numeric descriptors of germination and radicle growth events. Another aspect of digital imaging is the possibility to determine the colour space of a two-dimensional seed surface. Experiments carried out on lentil seed germination have shown that quantitative changes in Red-Green-Blue (RGB) colour component density may be considered as markers of the start of germination. In addition, the extracted RGB data may be used to trace a virtual three-dimensional surface plot allowing a better analysis of colour distribution on the lentil's surface. RGB colour density can also be used to determine any variation in colour due to the 'browning effect' as a result of advancing seed deterioration. The potential of RGB markers in classifying sub-samples and maintaining high germination quality in aged seed samples represents a non-destructive method in seed testing and sorting. As a conclusion, the information flow deriving from digital image processing should be integrated with other bio-morphological, taxonomic and 'omic-system' databases. The final target should be an interrelated and complex database for a deeper functional and structural knowledge of plant species, which can respond to the needs of farmers, seed industries, biodiversity conservation and seed basic research.
\end{abstract}

computerised image analysis / seed shape and size descriptors / seed colour components / seed testing and sorting

\section{INTRODUCTION}

Modern farming reflects a highly complex activity, where gains in crop yield depend directly on the continuous supply of energy and resources, such as intensive mechanisation and the development of agrochemicals to fertilise crops and control both weeds and pests, and crop selection versus monocultures that substitutes traditional varieties of agro- and eco-types. Therefore, when examining these problems it is impossible to separate the development of a more self-sustained agriculture by agroecosystems, which practise "ecological agriculture", from the modern strategy of "precision agriculture" which provides tools for reducing input costs, increasing yields and reducing environmental impacts in order to make decisions associated with crop production (Altieri et al., 1983; Cox, 2002).

\footnotetext{
* Corresponding author:

dellaquilaantonio@tiscali.it; (former Senior Scientist of the Institute of Plant Genetics - CNR, Bari, Italy).
}

In agroecology control, the remote-sensing techniques applied to classifying different cultivation area boundaries use digital imaging and the colour space value ratio (Peña-Barragán et al., 2008). Information technologies also include the development of digital image processing, focused mainly on cultivar identification (Keefe and Draper, 1986) and crop grading (Anquar et al., 2001), weed detection in seed samples (Granitto et al., 2002) or in open fields (Pérez et al., 1997), and on seed quality testing and sorting (Dell' Aquila, 2007). In this last case, the needs for development and improving seed quality tests are various, as reviewed by Hampton (1995). Farmers require information to expect rapidity and uniformity of seedling emergence, and seed industries need improved specific tests to guarantee the best levels of seed quality for production and trade purposes. Seed banks, devoted to ex situ conservation of biodiversity, require routine germination testing procedures before rejuvenating high quality accessions conserved in cold storage rooms, with the main target of avoiding 
plant genetic erosion and contributing to agroecosystem maintenance (Clergue et al., 2005). Furthermore, seed analysts and researchers are interested in developing new automated and non-destructive techniques to characterise seeds with welldefined genetic and physiological quality traits and improve knowledge in seed biology research.

Automated computer methods which utilise high-speed image capturing and data processing are the most advanced methods providing a high degree of accuracy in seed quality testing and sorting. Interest is increasing in the development of machine vision systems to replace human visual inspection, usually employed in germination tests under the rules of the ISTA (2005) and AOSA (2000). In analysing growth, the seed analyst studies rates of biological change with subjective methods. The germination test results essentially in a yes or no question, and the inspection of normal or abnormal seedlings at the end of the test depends on seed analysts' expertise. In addition, timing of the start of germination for individual seeds within a seed population is not accurately recorded because of the limited working time of the operator. To overcome these problems, many researchers have designed a number of machine vision system prototypes for the inspection of germinating seeds through the introduction of new image analysis parameters which can correlate biological changes more accurately and objectively. The understanding of growth rate patterns by image analysis parameters could represent a new method to investigate germination performance of many crop seed species in an automated way. As a result, new algorithms and hardware architectures have to be developed for high-speed extraction of raw data, such as seed digital images, and numeric data, such as dimensional measurements, shape factors and colour space density. Integration of image analysis data of seed germination performance with genomic and proteomic data could contribute to a better definition of the information technology' which accounts for acquisition, recording and elaboration, and communication of information.

This review reports features of image analysis system prototypes specifically designed for and widely applied in seed germination and vigour testing. The development of these technologies shows their potential in seed science study with the perspective of integrating traditional methods in seed quality assessment with those that provide more accurate and informative bio-morphological data.

\section{IMAGE PROCESSING IN SEED GERMINATION TESTING}

\subsection{Seed dimension and shape changes during imbibition: searching for a pattern}

Seeds have a three-dimensional (3-D) shape, while captured images displayed on the monitor or on a printed page are in two-dimensional (2-D) format (Loomis et al., 1999). In this case, the digital seed images can be assumed as a 2-D object having both dimensions placed along the orthogonal axes of a Cartesian plane. One way to describe the growth of a biological structure is by the velocity at which its points move, with
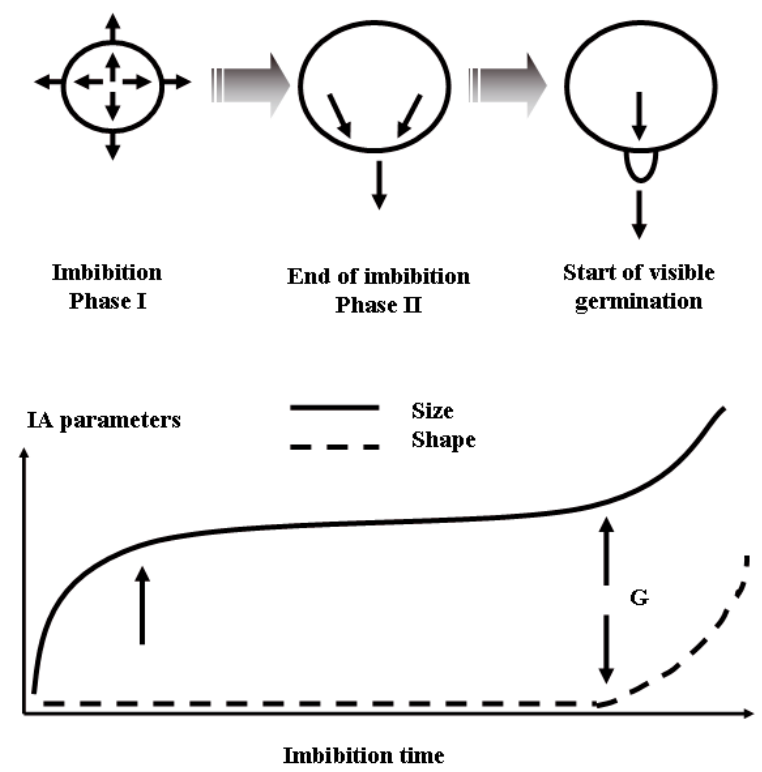

Figure 1. Note the changes in velocity fields (on the top) for an imbibing object-seed during the imbibition Phase I, at the end of imbibition Phase II, and at the start of radicle emergence from the seed coat. The related plotting of size or shape image analysis parameters versus imbibition time (on the bottom) shows curvature and inflection points (arrows) which describe the seed imbibition process up to a visible germination $(\mathrm{G})$.

respect to a fixed system of coordinates (Coen et al., 2004). These ideal patterns can be represented by vectors, which form a mathematical vector field for an object in movement. In the case of the seed imbibition process (Bewley, 1997), the first phase of rapid water uptake can be described geometrically as an enlargement of a disk which reflects radially an isogonic growth, where each region grows at the same rate in all directions (Fig. 1). Consequently, velocity fields change this type of direction and depend on a single direction corresponding to the reference point. The second phase of seed imbibition represents the stage at which growth is preparing to change direction and finalises in the emergence of the radicle tip, that is the signal of the so-called 'visible germination'.

These geometric changes result in a dynamic model which is inclusive of physiological, biochemical and molecular processes (Prusinkiewicz, 2004). Developmental models are commonly represented by growth pattern plotting, in which metric measurements of a 2-D object size, e.g. area, perimeter, length and width, or shape numeric factors, e.g. roundness, calculated with the formula: perimeter ${ }^{2} / 4 \pi$ Area, and aspect, calculated with the ratio between the longer axis and the shorter axis of the ellipse equivalent to the seed area, are plotted against time units to give a polynomial curve and equation (Silk, 1984). In the case of a seed, the completion of the first and the second phases of imbibition is marked by two inflection points, the latter marking the change in dimensions of the seed-object due to radicle protrusion from the seed coat, and coinciding with the time of germination completion or start of visible germination, in physiological and agronomic terms, respectively (Bewley, 
1997). When the shape change descriptors are used, during the two early phases of water uptake no apparent change in shape occurs, while curvature starts when the radicle tip protrudes from the seed coat with the related change in the shape of the seed-object. In fact, assuming that a seed with a circular shape has a roundness factor of 1 at the start of imbibition, a different shape of the seed, due to changes occurring with radicle protrusion from the seed coat, should produce a roundness factor higher than 1 (Dell'Aquila, 2004a). The inflection point of the related curve marks the start of visible germination.

\subsection{The case of Brassica seed germination: a model for image analysis application}

The most studied seeds are those of the Brassica genera because their morphology and shape are suitable for image analysis measurements. Assuming that a seed of cauliflower (Brassica oleracea L.) or broccoli (Brassica oleracea L.) approximates a sphere and that linear expansion during the first phase of water uptake is similar along both Cartesian coordinates, measurements of size and shape changes can be closely correlated with the increase in seed fresh weight, as previously reported (McCormac and Keefe, 1990; Dell'Aquila et al., 2000). Similar results were also obtained with seeds of winter wheat (Triticum aestivum $\mathrm{L}$.) and oat (Avena sativa $\mathrm{L}$.), with a shape differing from a sphere. Cereal seeds with 9.5$10.5 \%$ moisture content were subjected to a 'moistening' procedure to gradually reach $21-22 \%$ moisture content or to a 'drying' procedure to reach the original hydration state (Kruse, 2000). Using a machine vision system, the results showed that seed length, width and thickness increased linearly with moisture content with approximately the same proportionality. These basic findings allowed the design of a computeraided image analysis system (Fig. 2A), devoted essentially to monitoring seed image features during swelling and subsequent germination, operating in the laboratory of image analysis of the Institute of Plant Genetics of the National Research Council (IGV-CNR, Bari, Italy). A thermostatic chamber at $25^{\circ} \mathrm{C}$ was designed to include a colour Charged-Coupled Device (CCD) camera (Micropublisher 3.3M pixels, Qimaging, Canada), a timer-dependent lighting system, and a holder for a Petri dish containing polymerised agarose where a sample of a maximum of twelve Brassica seeds could be placed. Alternatively, agarose was substituted with highly concentrated $\mathrm{NaCl}$ for salt stress imbibition trials (Dell'Aquila, 2003), or different temperature regimes were used to evaluate their effects on germination performance (Dell'Aquila, 2005). The computer unit was standardised using a CCD camera with a 55-mm telecentric lens or a flat-bed scanner (Sharp mod. JX-330, Japan) for image capturing, a commercial imaging board, and a high-power personal computer running $\mathrm{MS}^{\circledR}$ Windows XP. The most recent version of the commercial software package ImagePro-Plus ${ }^{\mathrm{TM}}$ (IPP; Media Cybernetics, USA), or alternatively the open source freeware software ImageJ (http://rsb.info.nih.gov/ij/) were used for digital image processing. Time-lapse seed images were captured every hour in an automated way and saved on the hard disk in the most
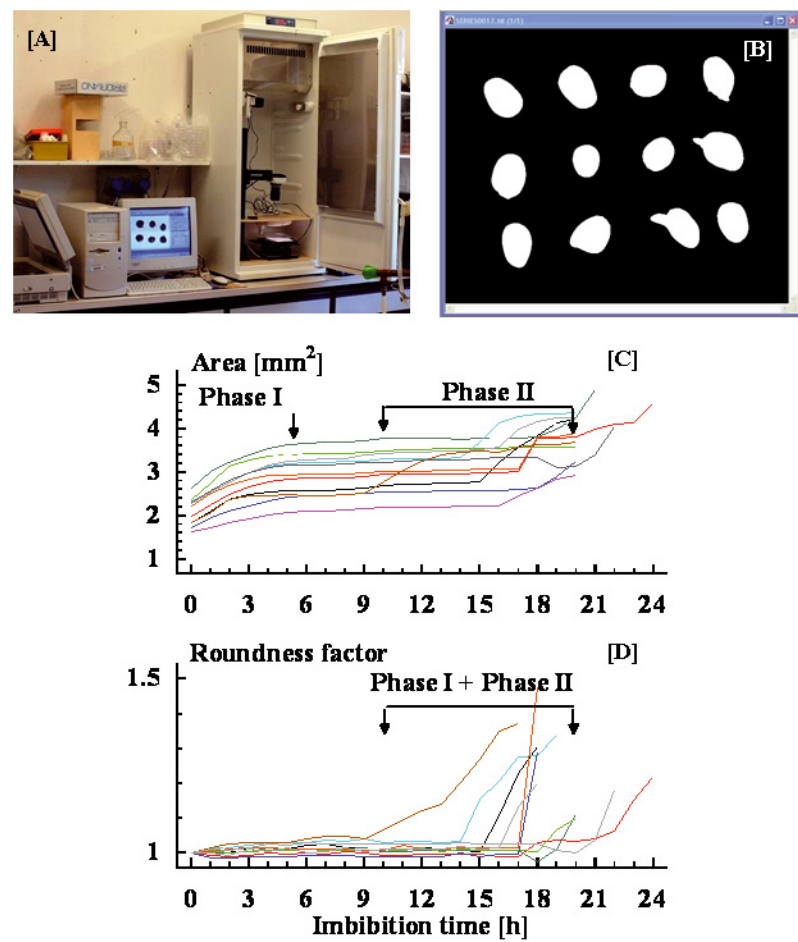

Figure 2. The machine vision prototype (A) operating in the IGVCNR (Bari, Italy) has been designed to capture digital images of Brassica seeds and to produce seed silhouettes (B) by image segmentation processing. Image analysis software can measure seed size and shape parameters, and data can be plotted to obtain, e.g., time courses of area (C) and roundness factor (D) increase. Arrows indicate: in (C), the end of Phase I ( $6 \mathrm{~h})$ and that of Phase II of area increase coinciding with the start of germination (11-21 h), respectively, and in (D), the end of Phase I + Phase II coinciding with the start of germination $(11-21 \mathrm{~h})$ as detected by the increase in the roundness factor (adapted from Dell' Aquila, 2004a).

common graphic formats with a full colour option. Image segmentation was carried out to streamline the process of object contour identification and to define the silhouette of the seeds (Fig. 2B). Image segmentation is the process of grouping pixels of a given image into homogeneous regions with respect to the contour of the object and the surrounding background, i.e. the inert support where seeds were placed. We used the multithresholding algorithm, which is able to differentiate the 2-D colour pair histograms (i.e., RG, RB, GB segmentation maps), giving a comprehensive segmentation map (Kurugollu et al., 2001). A macro was created with Image-pro's macro language (IPBasic, Media Cybernetics, USA) for the conversion of pixels into millimetres, object number counting, image analysis parameter measuring and data transfer to a MS ${ }^{\circledR}$ Excel worksheet.

Image analysis systems have been applied by several researchers to automatically test germination percentage in a large population of seeds. Van der Heijden et al. (1999) used a system controlled by a single computer program to study the germination time course in tomato (Lycopersicon esculentum L.), lettuce (Lactuca sativa L.), Arabidopsis and Brassica 
seeds under different temperature and water stress conditions. A more sophisticated image acquisition system was developed to capture images of different trays, containing plugs in which lettuce, cauliflower and tomato seeds were grown for subsequent transplanting (Ureña et al., 2001). Once the CCD camera was positioned over a given tray, a label placed on the tray surface containing the serial number in bar code form was read. Then, an image of the tray was obtained, allowing the examination of the cells to search for germinated seeds; the degree of seedling development was classified using fuzzy logic, and processed data on germination percentage and length of each seedling represented indices of speed of germination. More recently, Ducournau et al. $(2004,2005)$ elaborated new algorithms based on the idea that the emergence of a radicle tip at a defined time results in a modification of the binary images. The system was tested to study germination of sunflower (Helianthus annuиs L.) seeds, and detailed germination curves were obtained, allowing a perfect fit in a probit model (Ellis and Roberts, 1981).

\subsection{Information flow generated by the computer imaging process}

The image analysis system designed at IGV-CNR can be applied to study the imbibition process in several seed species and to set up new markers of seed germination and radicle growth rate. In this context, the generated information flow can be summarised as follows:

(1) Time-lapse seed images in the most common graphic file formats [usually Joint Photographic Experts Group (JPEG)] or Tagged Image File (TIF)] can be easily stored in the hardware to implement bio-morphological databases of plant species. The image sequencing can be assembled in a digital animation file format [usually Audio Live Interleave (AVI)] which can simulate in a short time the germination behaviour of a seed species. Digital seed catalogues or image libraries, used to study botanical structures for educational training as well as to share images via the Internet, have been provided by the Ohio State University, USA (Loomis et al., 1999; McDonald et al., 2001; http://www.cse.ohio-state.edu/\$ $\$$ sim\$fujimura/seed/).

A database was also developed at IGV-CNR to store information including images of several crop seeds, their germination simulation and elaboration of image analysis features collected from a 2-D imaging system. These data have been published on the following website: http://germimaging.ba.cnr.it for free download and educational purposes.

(2) A number of image analysis parameters for a single seed within a seed population may be extracted, including: metric size change descriptors (area, perimeter, length and width), shape change numeric factors (roundness and aspect), and surface colour space values (Red, Green, Blue colour primaries, and medium grey level density). Commercial or open source image analysis software packages can offer a number of algorithms to measure directly dimensions, shape and colour density of the desired object, and to combine these to calculate more complex parameters (Sundblad et al., 1998).

(3) Recent computer technology has developed powerful hardware and software suitable for elaborating in real time a large amount of data for statistical and graphical processing. As an example, data elaboration allows one to plot image analysis parameters (e.g., seed area and roundness factor) versus imbibition time, generating time-course patterns with curvature and inflection points for each individual seed (Fig. 2C, D). In highly viable cauliflower seeds a large variation in the time of the second phase of area increase contributed to different timing of the start of germination. When the imbibition process was monitored by the roundness factor, a first phase of no apparent shape change from the start of imbibition to radicle emergence was followed by a second phase of rapid increase with distinct curves. Image analysis was also applied to seed samples with different viability. The sensitivity of the technique in discerning the occurrence of the third phase of seed area increase or the second phase of roundness factor increase in deteriorated seeds, as well as in seeds subject to salt or temperature stress, provided a further test of the technique's flexibility (Dell'Aquila et al., 2000; Dell'Aquila, 2003, 2005). Seed area and roundness factor have been tested as effective markers of seed swelling and start of germination, with the advantage of being measured on each individual seed within a seed population (Dell'Aquila, 2004b). When immaturity, storage conditions or pathogen contamination affect the quality of a seed lot, individual seeds are not damaged to the same extent, resulting in the occurrence of an ample range of quality, possibly with distinct sub-populations. Computerised image analysis is a promising technique to investigate and detect any seed-to-seed variation within an apparently homogeneous population.

(4) The rate of rapid area or roundness factor increase in the last phase of imbibition, mostly due to protrusion of the radicle tip and its growth, may be correlated with the corresponding metric measurement of radicle length of a single germinated seed, when 'visible germination' can be assessed in a germination test. Highly significant correlation coefficients have been obtained in Brassica, radish (Raphanus sativus L.), lentil (Lens culinaris Medik.), lettuce, pepper (Capsicum annum L.), tomato and carrot (Daucus carota subsp. sativa L.) seeds (Dell'Aquila, $2004 a, b)$. In this way, the radicle elongation rate may be assessed indirectly by the corresponding seed area or roundness factor increase rates, confirming the usefulness of image analysis parameters in seed vigour assessment.

A promising field of application of image analysis is seed vigour testing, using a scanner image capturing technique. Sako et al. (2001) obtained digital images of lettuce seedlings, and the extracted image measurements were used to generate a vigour index based on morphological features. A similar method was also developed to assess vigour of different lots of small-sized horticultural seeds (Geneve and Kester, 2001; Oakley et al., 2004). Moreover, in cotton (Gossypium 
hirsutum L.), the problem of overlapping seedlings that can interfere with the measurements of elongation rate has been overcome with the design of a new algorithm which measures each seedling independently (Xu et al., 2007). Readers can find more information on the application of computer image analysis in seed vigour testing in the recent review of Dell' Aquila (2007).

\section{RED-GREEN-BLUE COLOUR SPACE EVALUATION IN SEED DIGITAL IMAGES}

\subsection{The case of lentil seed: from two-dimensional digital imaging to three-dimensional surface simulation}

Physiological studies have demonstrated that there are only three colour sensors utilised by human visual recognition, which are associated with long (Red, R), medium (Green, G) and short (Blue, B) wavelengths of light (Fairchild, 1998). All visible colours, as displayed on most computer monitors, can be represented by varying combinations of these primaries. Even if colour mapping is defined by a numeric range of RGB values (from 255-255-255 for the so-called 'white' colour to 0-0-0 for the so-called 'black' colour), many colour models are used to represent colour space with other primaries, such as hue, saturation, intensity value (HSV), or hue, saturation and lightness (HSL), or cyan, magenta and yellow (CMY). To study colour features of seed digital images we used the RGB colour order system by extraction of any colour information whose appearance is generally altered by surface texture, lighting, shading effects and viewing conditions. By automated image analysis all these factors must be considered in the characterisation of a coloured image of a seed through a series of colour histograms. One of these may be graphically displayed on a Cartesian plane by extracting the number of pixels of each primary colour along a virtual line connecting the seed grain and emerging radicle tip. In the visualisation scheme of Figure 3 the implemented algorithms follow a similar progression: (1), selection of RGB colour space; (2), quantification of RGB pixel values; and (3), computation of histograms by plotting RGB data (Y-axis) versus distance (X- axis) reported in metric units. Otherwise, we can consider an unlimited number of colour histograms placed on a plane (X-and Y-axes) with combinations of RGB primary pixel values (Z-axis). The extracted data of coordinates and primary colour density can be integrated with optional data, useful for enhancing the rendering performance of a 3-D seed surface simulation. In addition, the choice of visualisation mode by surface dots, lines, meshes and filled surface may help a better plotting of the 3-D surface of the studied seed.

As an example, RGB data, extracted along the virtual straight line connecting the radicle tip and the corresponding free border of a lentil seed coat, may be used to plot the related histogram (Fig. 4). Before radicle emergence (14 h of imbibition, Fig. 4A, B) RGB density distribution is representative of the seed coat colour space which is limited by $0-0-0$ pixel values corresponding to the black background holder where seeds

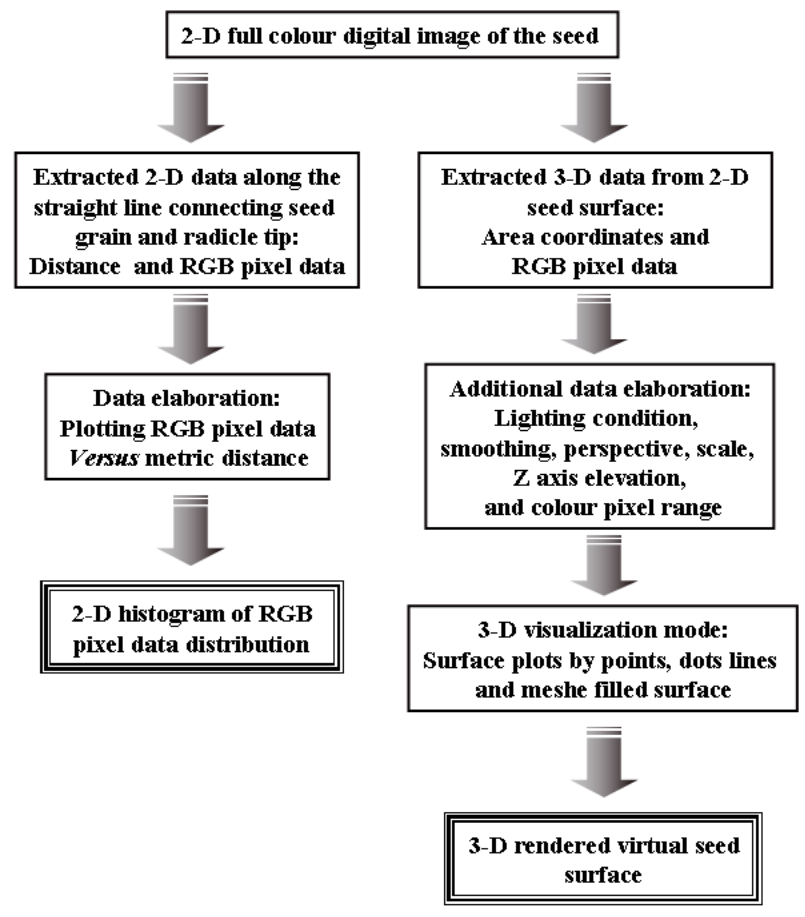

Figure 3. Digital seed image processing scheme by Red-Green-Blue (RGB) value computation. On the left, the image processing flow shows that extracted two-dimensional (2-D) data from the digital image of a seed surface can be computed to generate RGB histograms by plotting colour components (Y-axis) versus distance (X-axis). On the right the data elaboration flow is reported, which takes into account an unlimited number of RGB histograms distributed on a plane (Xand Y-axes) with combinations of RGB primary pixel values (Z-axis). The extracted 2-D data from the seed surface can be further processed using optional tools useful for enhancing the rendering performance of a three-dimensional (3-D) seed surface simulation.

are placed. At $15 \mathrm{~h}$ of imbibition (Fig. 4C, D), the start of radicle protrusion from the seed coat can be evidenced by a dropping point between the RGB distribution of the seed coat and that corresponding to the emerging radicle tip. Timing of the occurrence of this inflection point marks the start of 'visible germination'. At $21 \mathrm{~h}$ of imbibition (Fig. 4E, F), when radicle length reaches $2.5-3 \mathrm{~mm}$, the RGB component histogram clearly shows two sequentially different distributions of RGB colour primaries due to the seed grain and developed radicle with a brown or white-yellow colour, respectively. The 3-D rendering option lets us obtain a 3-D surface plot of a lentil seed at 14,15 and $21 \mathrm{~h}$ in mesh mode using the plug-in 'Interactive 3D surface plot' of the image analysis software ImageJ (Fig. 5). By changing the modality of smoothing and Z-axis elevation modality, surface features can be enhanced and certain quality factors such as seed coat rugosity, disease and defects can be determined quantitatively using the facilities of image analysis processing.

More recently, a new technology has been developed known as dynamic speckle, or biospeckle, an optical phenomenon produced when living materials, such as biological tissues, are illuminated by laser light. The term speckle refers to a random 

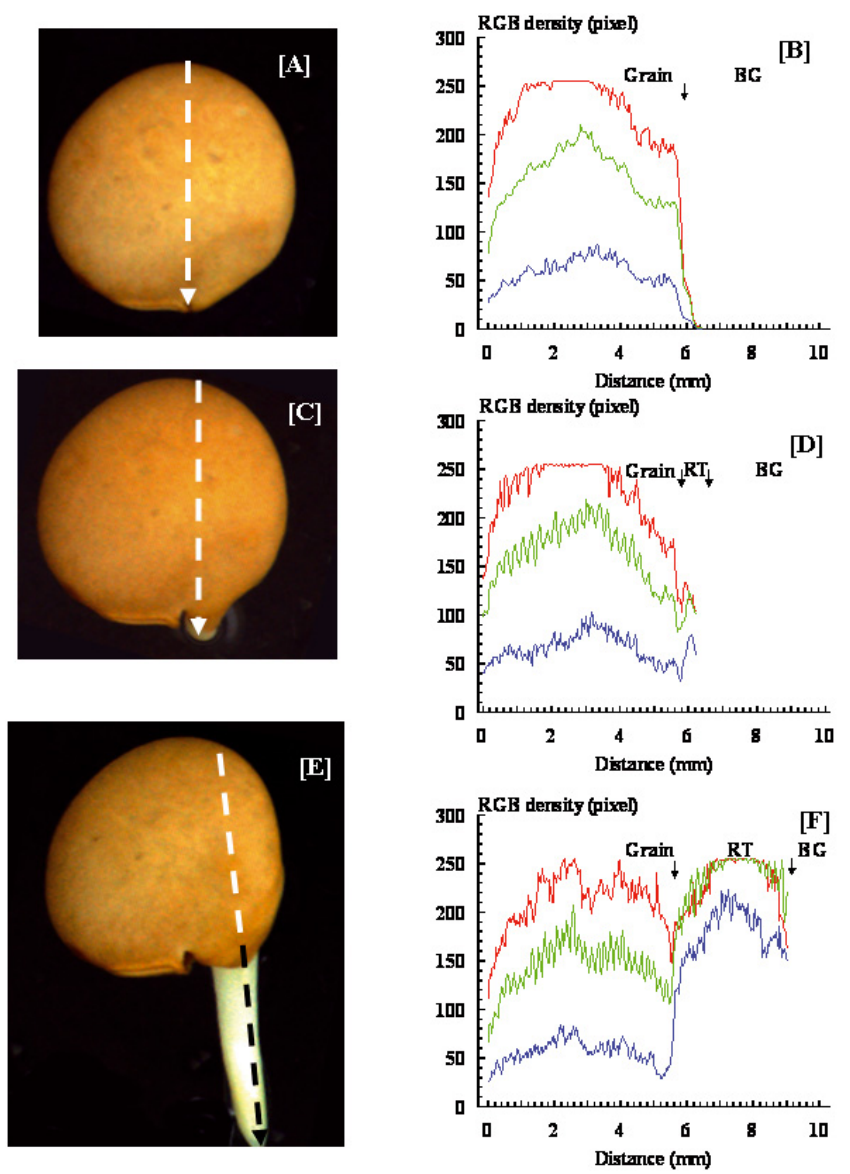

Figure 4. Seed images, captured by a Charged-Coupled Device camera at $14(\mathrm{~A}), 15(\mathrm{C})$ and $21 \mathrm{~h}(\mathrm{E})$, can be a source of two dimensional imaging data along the straight dashed line connecting the seed grain and radicle tip (RT). Related Red-Green-Blue (RGB) density values are plotted as histograms (B, D and F, respectively) in a Cartesian plane having the metric distance as X-axis and RGB density as Y-axis (adapted from Dell'Aquila, 2007). BG, background.

granular pattern which can be observed when a highly coherent light beam is diffusely reflected on a surface with a complicated structure. Features of seed tissue images, acquired by a CCD camera, can be amplified and assessed by their speckle activity by appropriate algorithms (Braga et al., 2003). The biospeckle technique has proved to be a potential nondestructive methodology to assess bean (Phaseolus vulgaris L.) seed viability, even if water content in the seeds can affect measurements. The technique has also been applied to detect the presence of fungi colonies on bean seed coats (Braga et al., 2005).

\subsection{Red-Green-Blue data as markers of seed viability}

Object surface colour space is a physical feature which can be used for effective quantitative recognition during image analysis processing. The experimental approach extracts automatically the colour content of a restricted or entire space of
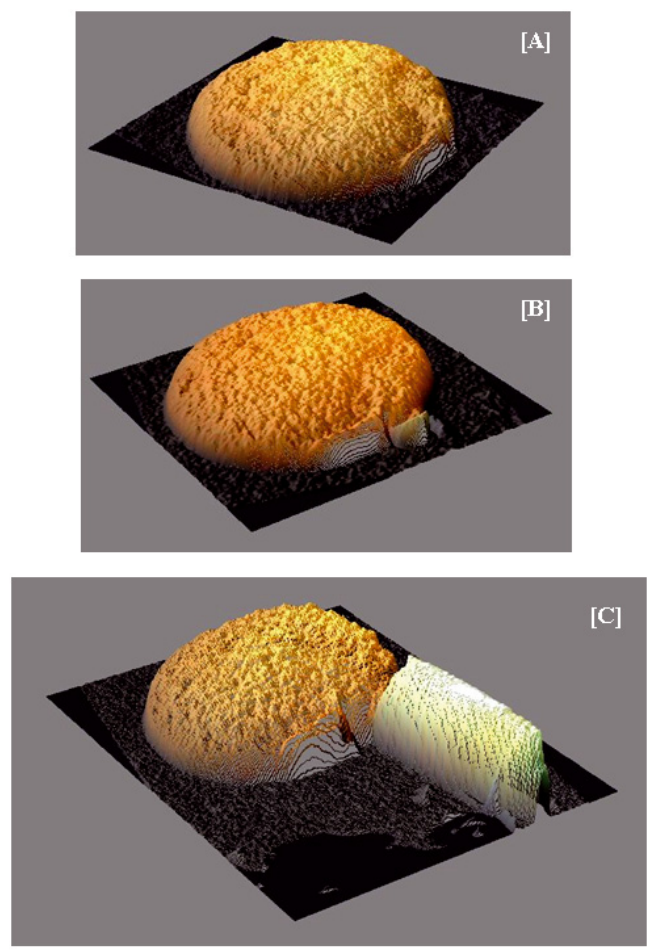

Figure 5. Three-dimensional (3-D) rendering option is used to obtain a virtual 3-D surface plot of lentil seeds at 14 (A), 15 (B) and $21 \mathrm{~h}$ (C). The elaboration is made in mesh mode by the interactive 3-D surface plot plug-in of the ImageJ software package.

the seed surface over a large collection and sequence of images. The Red, Green and Blue colour components alone can be quantified as medium density for a normalised seed area, and so data can be easily compared and used for cluster or discriminating analysis. We analysed RGB colour components on lentil seed samples stored under deteriorating conditions to investigate the potential of this image analysis parameter to be a valid seed viability marker (Dell'Aquila, 2006). Images of differently deteriorated lentil seed samples were captured by a flat-bed scanner with full colour option after a calibration with Kodak Q-60 target to ensure an accurate transposition of dye densities to RGB pixel values. The density values of Red, Green and Blue colour primaries, or alternatively a medium RGB value corresponding to the density colour of a grey image, were determined using the software package IPP v. 6.01. RGB density distribution for each seed sample was graphically displayed by defining equal classes of pixel unity on the $\mathrm{X}$-axis and by plotting the number of seeds in each class on the Y-axis. Three fractions can be extracted, whose borders were chosen so that two fractions (with high and medium colour density) contained seeds with high viability and the third fraction, with low colour density, the rest of seeds, according to the method used in sorting cabbage seeds with a chlorophyll fluorescence marker (Dell' Aquila et al., 2002).

A sequence of seed images may be acquired during different periods of ageing under different environmental storage conditions. Colour differences between deteriorated seeds can 



Figure 6. The "browning effect" due to deterioration (det) is shown in the captured digital images of lentil seeds stored under $14.4 \% \mathrm{mc}$ and $40{ }^{\circ} \mathrm{C}$ conditions for 0 (A) and $51 \mathrm{~d}$ (B; adapted from Dell'Aquila, 2006). The extracted Red-Green-Blue (RGB) colour space data from two-dimensional imaging are used to plot seed number percentage versus the changes in colour primary component density at 0 and $51 \mathrm{~d}$ of deterioration (C). The arrows indicate the selected border of each fraction (FI, FII and FIII, respectively) for each colour component, having different viability patterns during advancing ageing.

be due to accumulation of Amadori and Maillard products, obtained by reduction of sugars or protein aminogroups to form fructosyl derivates or glycate proteins, whose interaction produces polymeric brown products (Wettlauer and Leopold, 1991; Sun and Leopold, 1995). The effect has been described in legumes, where colour change can be quite heterogeneous within a seed sample and seeds which maintain their original colour at full maturity tend to preserve high vigour (Priestley, 1986). As a result, the visible physical change is the discoloration or browning of the seed coat, as shown for lentil seeds stored over 51 days at $14.4 \%$ moisture content and $40{ }^{\circ} \mathrm{C}$ (Fig. 6A, B). Based on the symmetric distribution of density values for each colour component in lentil seed samples aged for both 0 and 51 days, the three seed fractions can be defined with different RGB value borders (Fig. 6C). Alternatively, it is possible to use an overall medium RGB index (255 grey levels) in both seed samples with different value borders (Dell' Aquila, 2006). At $0 \mathrm{~d}$ deterioration Fraction I contained few seeds
$(1.33 \%)$ with $78 \%$ final germination $(\mathrm{G})$, while at $51 \mathrm{~d}$ deterioration a large amount $(44.16 \%)$ of seeds was found with the lowest germination percentage $(25 \% \mathrm{G})$. Conversely, in Fraction III at 0 d deterioration more seeds $(61 \%)$ had $98 \%$ G compared with $51 \mathrm{~d}$ deteriorated ones $(10.6 \%$ in number and $75 \%$ G). The largest amount of seeds was found in Fraction II from both 0 and $51 \mathrm{~d}$ deteriorated seed samples (76.5 and $54.16 \%$, respectively, with 39 and $55 \% \mathrm{G}$, respectively). The RGB marker was also used in sorting deteriorated seeds of cucumber (Cucumis sativus L.), lettuce and tomato (Dell'Aquila, 2007). These findings confirmed that a RGB marker may identify seed sub-groups with different germination quality and variable seed distribution using a non-destructive technique. Collected deteriorated seed images from different species together with RGB threshold values can be stored in an electronic archive and constitute a database of deterioration patterns useful for elaborating a strategy of highly viable seed sorting and survival prediction.

\section{PERSPECTIVE OF IMAGING INFORMATION TECHNOLOGY AS A TOOL IN SEED SCIENCE AND TECHNOLOGY}

In the 21st century, plant researchers have a range of opportunities provided by technological and knowledge advances. Many of these tools can be considered under a unique subject, 'information technology', which by definition is concerned with the management of biological system databases (Cox, 2002). More recently, inspection of plants utilises modern image acquisition aided by computer technology, providing a highly adaptable tool to produce digital images suitable for subsequent processing. Automated image analysis systems applied to the study of seed quality provide a semi/non-destructive evaluation tool of seed performance leading to germination, or in the dry state an estimate of the morphological features useful for seed testing and sorting. As a result, time-course patterns can give information on hydration status or colour space quality. In addition, medium density of RGB colour primaries can be used as a non-destructive marker in classifying seeds with different viability levels within a seed population. These findings are in agreement with the recent success of optical methods which incorporate highspeed optical sensing and data processing techniques to facilitate quality evaluation and sorting of many agricultural products with a high degree of accuracy (Chen and Sun, 1991). The success of this new technology has also been supported by the declining costs of computer hardware and the opportunities to acquire image analysis and graphical software packages with new algorithms designed to implement the capability of data processing and plotting.

Both digital imaging and numeric data acquisition and elaboration, as well as their utilisation, should be aimed at integration with more complex databases. The final target of information technology is that any database is transferred in the right way to implement other databases, e.g., those generated by so-called 'omic systems', for an up-to-date knowledge of plant functional and structural changes and modelling. As 
an example, we have calculated that in the case of a sample of twelve broccoli seeds' imbibition at $25^{\circ} \mathrm{C}$ for $24 \mathrm{~h}$, the extracted data could be quantified in 288 single seed images and 2.592 image analysis numeric data (Dell'Aquila, 2007). This amount of data, the minimum needed to describe broccoli seed imbibition and germination, may be further processed statistically and displayed graphically. The creation of an extensive database could allow matching of different bio-morphological characteristics of a large number of seed species to improve genetic purity analysis, taxonomy screening, germination and vigour prediction. Keys (1982) first developed a computerised automated seed analysis system in order to enable analysts to estimate physiological parameters of seeds and compare the values obtained with the standards for seeds of known quality. Many years later, an image-based database was designed at the National Seed Storage laboratory of USDA-ARS (Fort Collins, USA), with the aim of concentrating data of seed physical characteristics and, so, to enhance conservation and utilisation of seed germplasm (Howarth and Stanwood, 1993). A 'Seed Identification Key' using a computerised database, also available commercially in a CD format, has been developed to identify prohibited and restricted seeds and to reduce the impact of noxious weeds on the environment and agricultural production (Gupta et al., 2005). Daoust et al. (2005) described a machine learning technique that employed the use of an inexpensive commercial scanner and a modern PC. The image analysis software used allowed the definition of 21 seed species with the digitised images and the measurement of size parameters and average colour on each seed. The classification determined the closest matching species for each seed using a global database highly configurable to specific seed types.

\section{CONCLUSION}

An information system consists of two components: a computer (images, data, information processing engine) and human-computer interaction (Lew et al., 2007). In the case of seed inspection by a vision machine system, the overall goal is to extract from a two-dimensional digital image a considerable amount of data in order to describe germination and radicle growth, and the colour space density of the seed surface. The sophistication of non-destructive methods has evolved rapidly, and the availability of high-speed data acquisition and processing technology has encountered a renewed interest in seed researchers. The up-to-date designed machine vision systems are prototypes which need to be highly automated with the implementation of new algorithms, so that valid and flexible image analysis parameters can integrate or substitute the visual inspection of a large seed sample for germination and vigour testing. Efforts should be made in the future to utilise this kind of information technology, which can be included in the global key to precision agriculture and sustainability, in transferring standardised data to a seed analyst for decision-making or recording purposes.

Acknowledgements: The present research work was partially performed with the aid of the 'Short Mobility Programme' of the Italian National Research Council (CNR) during the years 1999-2004.

\section{REFERENCES}

Altieri M.A., Letourneau D.K., Davis J.R. (1983) Developing sustainable agroecosystems, Bioscience 33, 45-49.

Anquar F., Mannino M.R., Casals M.L., Fougereux J.A., Demilly D. (2001) Carrot seeds grading using a vision system, Seed Sci. Technol. 29, 215-225.

AOSA (2000) Rules for testing seeds, in: Association of Official Seed Analysts (Eds.).

Bewley J.D. (1997) Seed germination and dormancy, Plant Cell 9, 10551066.

Braga R., Dal Fabbro I.M., Borem F.M., Rabelao G., Arizaga R., Rabal H., Trivi M. (2003) Assessment of seed viability by laser speckle techniques, Biosyst. Eng. 86, 297-294.

Braga R., Rabelo G.F., Granato L.R., Santos E.F., Machado J.C., Arizaga R., Rabal H.J., Trivi M. (2005) Detection of fungi in beans by the laser biospeckle technique, Biosyst. Eng. 91, 465-469.

Chen P., Sun Z. (1991) A review of non-destructive methods for quality evaluation and sorting of agricultural products J. Agric. Eng. Res. 49, 85-98.

Clergue B., Amiaud B., Pervanchoon F., Laserre-Joulin F., Plantureux S. (2005) Biodiversity: function and assessment in agricultural areas. A review, Agron. Sustain. Dev. 25, 1-15.

Coen E., Rolland-lagan A.-G., Matthews M., Bangham J.A., Prusinkiewicz P. (2004) The genetics of geometry, PNAS $101,4728-4735$.

Cox S. (2002) Information technology: the global key to precision agriculture and sustainability, Comp. Electron. Agricult. 36, 93-111.

Daoust T., Fujimura K., McDonald M.B., Bennett M.A. (2005) A computer-based system for seed identification, Seed Technol. 27, 190-202.

Dell'Aquila A. (2003) Image analysis as a tool to study deteriorated cabbage (Brassica oleracea L.) seed imbibition under salt stress conditions, Seed Sci. Technol. 31, 619-628.

Dell'Aquila A. (2004a) Cabbage, lentil, pepper and tomato seed germination monitored by an image analysis system, Seed Sci. Technol. $32,225-229$.

Dell'Aquila A. (2004b) Application of a computer-aided image analysis system to evaluate seed germination under different environmental conditions, It. J. Agron. 8, 51-62.

Dell'Aquila A. (2005) The use of image analysis to monitor the germination of seeds of broccoli (Brassica oleracea L.) and radish (Raphanus sativus L.), Ann. Appl. Biol. 146, 545-550.

Dell'Aquila A. (2006) Red-Green-Blue (RGB) colour density as a nondestructive marker in sorting deteriorated lentil (Lens culinaris Medik.) seeds, Seed Sci. Technol. 34, 609-619.

Dell'Aquila A. (2007) Towards new computer imaging techniques applied to seed quality testing and sorting, Seed Sci. Technol. 38, $519-538$.

Dell'Aquila A., van Eck J.W., van der Heijden G.W.A.M. (2000) The application of image analysis in monitoring the imbibition process of white cabbage (Brassica oleracea L.) seeds, Seed Sci. Res. 10, $163-169$.

Dell'Aquila A., van der Shoor R., Jalink H. (2002) Application of chlorophyll fluorescence in sorting controlled deteriorated white cabbage (Brassica oleracea L.) seeds, Seed Sci. Technol. 30, 689-695.

Ducournau S., Feutry A., Plainchault P., Revollon P., Vigouroux B., Wagner M.H. (2004) An image acquisition system for automated monitoring of the germination rate of sunflower seeds, Comp. Electron. Agric. 44, 189-202.

Ducournau S., Feutry A., Plainchault P., Revollon P., Vigouroux B. (2005) Using computer vision to monitor germination time course of sunflower (Helianthus annus L.) seeds, Seed Sci. Technol. 33, 329-340. 
Ellis R.H., Roberts E.H. (1981) The quantification of ageing and survival in orthodox seeds, Seed Sci. Technol. 9, 373-409.

Fairchild M.D. (1998) Color appearance Models, Addison-Wesley, Reading, MA.

Geneve R.L., Kester S.T. (2001) Evaluation of seedling size following germination using computer-aided analysis of digital images from a flat-bed scanner, Hort. Sci. 36, 1117-1120.

Granitto P.M., Navone H.D., Verdes P.F., Ceccato H.A. (2002) Weed seeds identification by machine vision, Comp. Electr. Agr. 33, 91103.

Gupta M.L., George D.L., Basnet B.B. (2005) Seed identification using a computerised database, Seed Sci. Technol. 33, 647-654.

Hampton J.C. (1995) Methods of viability and vigour testing: a critical appraisal, in: Basra A.S. (Ed.), Seed Quality. Basic Mechanism and Agricultural Implications, Food Products Press, The Haworth Press, Inc. New York, pp. 81-118.

Howarth M.S., Stanwood P.C. (1993) Imaging techniques to enhance the preservation and utilization of seed germplasm J. Seed Technol. 17, 54-64.

ISTA (2005) International rules for seed testing, in: International Seed Testing association (Eds.).

Keefe P.D., Draper S.R. (1986) The measurement of new characters for cultivar identification in wheat using machine vision, Seed Sci. Technol. 14, 715-724.

Keys R.D. (1982) CASAS (computerized automated seed analysis system): an approach to the analysis and testing of seed J. Seed Technol. 7, 23-35.

Kruse M. (2000) The effect of moisture content on linear dimensions in cereal seeds measured by image analysis, Seed Sci. Technol. 28, 779-791.

Kurugollu F., Sankur B., Harmanci A.E. (2001) Color image segmentation using histogram multithresholding and fusion, Image Vision Comput. 19, 915-928.

Lew M., Sebe N., Huang T.S. (2007) The Age of human computer interaction, Image Vision Comput. 25, 1833-1835.

Loomis J.J., Fujimura K., McDonald M., James D., Bennett M. (1999) Using computer graphics for three-dimensional seed cataloguing, Seed Sci. Technol. 27, 439-446.

McCormac A.C., Keefe P.D. (1990) Cauliflower (Brassica oleracea L.) seed vigour: imbibition effects J. Exp. Bot. 41, 893-899.
McDonald M.B., Evans A.F., Bennet M.A. (2001) Using scanner to improve seed and seedling evaluations, Seed Sci. Technol. 29, 683689.

Oakley K., Kester S.T., Geneve R.L. (2004) Computer-aided digital image analysis of seedling size and growth rate assessing seed vigour in Impatiens, Seed Sci. Technol. 32, 837-845.

Peña-Barragán J.M., López-Granados F., García-Torres L., JuradoExpósito M., de la Orden M.S., García-Ferrer A. (2008) Discriminatin cropping systems and agro-environmental measures by remote sensing, Agron. Sustain. Dev. 28, 355-362.

Pérez A.J., López F., Benlloch J.V., Christensen S. (1997) Colour and shape analysis techniques for weed detection in cereal fields, First European Conference for information Technology in Agriculture, Copenhagen, 15-18 June, pp. 45-50.

Priestley D.A. (1986) Morphological, structural, and biochemical changes associated with seed ageing, in: Priestley D.A. (Ed.), Seed Aging, Comstock Publishing Associates, Ithaca and London, pp. 125-195.

Prusinkiewicz P. (2004) Modelling plant growth and development, Curr. Opin. Plant Biol. 7, 79-83.

Sako Y., McDonald M.B., Fujimura K., Evans A.F., Bennett M.A. (2001) A system for automated seed vigour assessment, Seed Sci. Technol. $29,625-636$.

Silk W.K. (1984) Quantitative descriptions of development, Ann. Rev. Plant Physiol. 35, 479-418.

Sun W.Q., Leopold A.C. (1995) The Maillard reaction and oxidative stress during aging of soybean seeds, Physiol. Plant. 94, 94-104.

Sundblad L.-G., Geladi P., Dunberg A., Sundberg B. (1998) The use of image analysis and automation for measuring mitotic index in apical conifer meristems J. Exp. Bot. 49, 1749-1756.

Ureña R., Rodriguez F., Berenguel M. (2001) A machine vision system for seeds germination quality evaluation using fuzzy logic, Comp. Electron. Agric. 32, 1-20.

van der Heijden G.W.A.M, Polder, G., van Eck J.W., Jalink H., van der Shoor R. (1999) Automatic determination of germination of seeds, 1999 Word Seed Conference, 6-8 September 1999, Cambridge, UK, Programme \& Abstract, p. 14

Wettlauer S.H., Leopold, A.C. (1991) Relevance of Amadori and Maillard products to seed deterioration, Plant Physiol. 97, 165-169.

Xu L., Fujimura K., McDonald M.B. (2007) Automatic separation of overlapping seedlings by network optimization, Seed Sci. Technol. $35,337-350$. 